\title{
Conjunctival Melanoma: A New Clinical and Therapeutical Approach
}

\section{Rodríguez-Martín ${ }^{\mathrm{a}} \quad$ J. Rodríguez-Martín ${ }^{\mathrm{c}}$}

\section{N. Merino de Paz ${ }^{\mathrm{a}} \quad$ P. Contreras Ferrer ${ }^{\mathrm{a}} \quad$ P. Rocha Cabrera \\ B. Rodríguez Martín ${ }^{d} \quad$ G. Gordillo Santana ${ }^{d}$}

\section{A. Martín-Herrera ${ }^{b} \quad$ A. Noda-Cabrera ${ }^{a}$}

Departments of a Dermatology, ${ }^{\text {bPathology, }}$ 'Ophthalmology, and d Nursery Department, Hospital Universitario de Canarias, University of La Laguna, La Laguna, Spain

\section{Key Words}

Melanoma $\cdot$ Therapeutics $\cdot$ Dermoscopy $\cdot$ Oncology

\begin{abstract}
Melanoma involving the conjunctiva is extremely rare. Graver prognosis has been reported with primary conjunctival melanoma than with their cutaneous counterparts [Collin et al.: Aust N Z J Ophthalmol 1986;14:29-34]. Among conjunctival melanomas, two significant risk factors for tumour-related death have been identified: (i) age older than 55 years and (ii) unfavourable tumour location (caruncle, cornea, fornix, palpebral conjunctiva) [Werschnik and Lommatzsch: Am J Clin Oncol 2002;25:248-255]. Here we present a rare case of lentigo maligna involving the palpebral, bulbar conjunctiva and the caruncle. We describe dermoscopic patterns observed and the use of a novel ocular melanoma therapy with topical imiquimod.
\end{abstract}

\section{Case Report}

A 74-year-old man was referred to our Department with an asymptomatic lesion located in the bulbar conjunctiva since childhood. He reported that the lesion had grown fast during the last months, presenting with diffuse pigment clusters involving the tarsal, bulbar and lower palpebral conjunctiva of the right eye and caruncle. The patient was a Fitzpatrick phototype II and his clinical history was relevant for multiple actinic keratosis and a parotideal epidermoid carcinoma.

On physical examination, the patient had several ill-defined, small brown and black macules located in the bulbar, palpebral and tarsal conjunctiva and the caruncle (fig. 1). The dermoscopic study showed peppering, globules and dots distributed in a parallel pattern in the tarsal and marginal conjunctiva and dilated vessels in the bulbar conjunctiva. Pigmented blotches in the caruncle were observed. 


\begin{tabular}{l|l|l|l} 
Case Reports $\boldsymbol{h}$ (Case Rep Dermatol 2010;2:149-155 & Published online: August 27, 2010 & $\begin{array}{l}\text { ○ 2010 S. Karger AG, Basel } \\
\text { ISSN 1662-6567 } \\
\text { www.karger.com/cde }\end{array}$ \\
$\begin{array}{ll}\text { Dol: } 10.1159 / 000320246 \\
\end{array}$ & & & \\
\hline
\end{tabular}

Polychromia was also evident, with the presence of black, light brown, dark brown and grey colours. Dilated vessels with a rope-ladder pattern in the palpebral conjunctiva were observed (ig. 2 , fig. 3 ). The parallel conformation found in our patient may correspond to the dendritic distribution of the melanocytes between the keratinocytes in the epithelium. The rope-ladder pattern observed in the palpebral conjunctiva could reflect the vessels crossing the dense fibrous stroma of the tarsus.

Incisional biopsies were performed. Microscopic review of the specimens demonstrated an atypical proliferation of melanocytes in a lentiginous pattern. Melanocytes showed a pagetoid distribution between the keratinocytes in the epithelium. Immunohistochemical stains were positive for S-100,

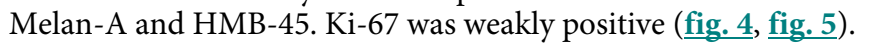

After histopathological confirmation of lentigo maligna (LM), the patient underwent treatment by excisional biopsy of the larger lesions and adjuvant cryotherapy resulting in no visibly apparent disease. Histopathological analysis of the specimen confirmed the previous diagnosis of LM. A total body $\mathrm{PET} / \mathrm{CT}$ scan was negative for distant metastasis. Routine blood tests were in normal ranges. These examinations resulted in the diagnosis of an AJCC-UICC stage of T1; N0, M0.

Due to the multifocal distribution of the tumour, adjuvant topical mitomycin $\mathrm{C}$ was added $(0.04 \%$, four times daily) during 3 months. Topical imiquimod (IMQ) $5 \%$ cream ( $250 \mathrm{mg}$ ) was started in the caruncle area once daily, five times per week. Weekly follow-up was achieved. The patient experienced temporal conjunctival and cutaneous irritation and eyelid oedema, but this was alleviated by artificial tears and lubricating ointment and the therapy was continued. No systemic side effects were noted. He completed a 6-week course of IMQ therapy that resulted in complete regression of the caruncle lesion. One year after completion of therapy, he remains disease-free clinically and pathologically ( $\underline{\text { fig. } 6})$.

\section{Discussion}

The decisive diagnosis procedure in conjunctival melanoma is the histological examination [1-4]. Dermoscopy is a noninvasive technique that can help us to distinguish between benign and malignant lesions. Histological findings and the differences between benign and malignant conjunctival lesions $[5,6]$, even the correlation with confocal microscopy $[7,8]$ have been described in the literature. Nevertheless, only one manuscript describes digital surface microscopy parameters to evaluate these lesions [7]. They suggest four categories for evaluating the lesions: geometry (area, maximum and minimum diameter), colour, texture and islands of colour. Higher values in geometry, darker colour and predominance of blue colours, higher contrast and the imbalance of blue-grey regions are suggestive of melanoma.

The three most common forms of melanoma described in the skin are: (1) LM, (2) superficial spreading melanoma, and (3) nodular melanoma. These forms can also be recognized in the conjunctiva. As in the skin, LM appears to be associated with a good prognosis compared to the prognosis associated with superficial spreading of melanoma. These two forms of melanoma are both associated with an intraepithelial stage and had previously been grouped under one designate, cancerous melanosis [6-9]. They can be distinguished histologically although definite clinical differentiation is to be determined in the future. Dermoscopy could add an interesting tool to this field.

Surgical excision is the gold standard for melanoma; however, because lesions are often quite large or involving sensitive areas such as the eyelid, extensive surgery could produce cosmetic deformity and functional disability $[8,9]$. Possible therapeutic approaches to this condition are exenteration of the orbit, excision combined with cryotherapy, and excision combined with radiotherapy [8]. Nonsurgical options include cryotherapy, topical mitomicin or interferon. IMQ is a topical immunomodulator available in a $5 \%$ cream that can generate a local cytotoxic response with potentially antiviral and antitumor effects. IMQ is approved for the treatment of genital warts, actinic keratosis and superficial basal cell carcinomas. Off-label uses include: mycosis fungoides, Bowen's disease and LM [10]. 
Topical IMQ 5\% cream is a valid option for patients with comorbid conditions, lesions located in sensitive areas or extensive lesions that may discourage surgery. Patients may experience constitutional symptoms, punctate keratopathy or diminished visual acuity as side effects associated with cytokine release, but these should resolve if the treatment is discontinued [11]. Although these results are relatively short-term, the findings suggest that IMQ topical therapy could be considered as an adjuvant therapy in cases of caruncle involvement in ocular LM and melanoma in situ. We consider IMQ as an option in the treatment of patients in whom surgery may be contraindicated or for those in whom the cosmetic or functional consequences would be considerable, like our patient.

Most importantly, the patient needs to be aware that it is an off-label use and the longterm effectiveness of this treatment has not been proven.

Fig. 1. Several ill-defined, millimetric brown and black macules located in the bulbar, palpebral and tarsal conjunctiva and the caruncle could be observed.

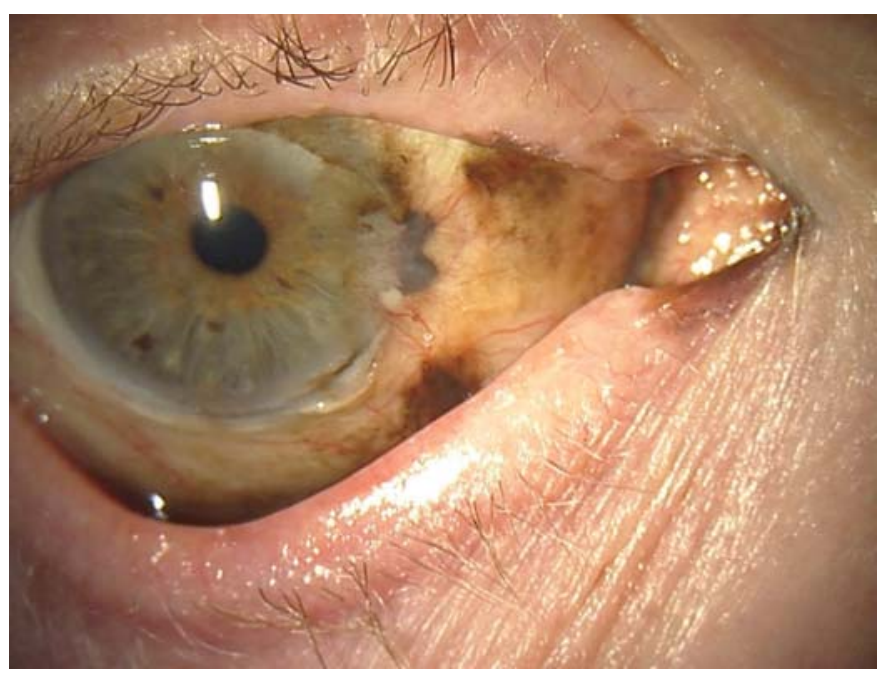


Fig. 2. Light and dark brown globules and dots could be observed in a parallel pattern. Brown blotches and arborizing vessels in the palpebral and bulbar conjunctiva were also found.

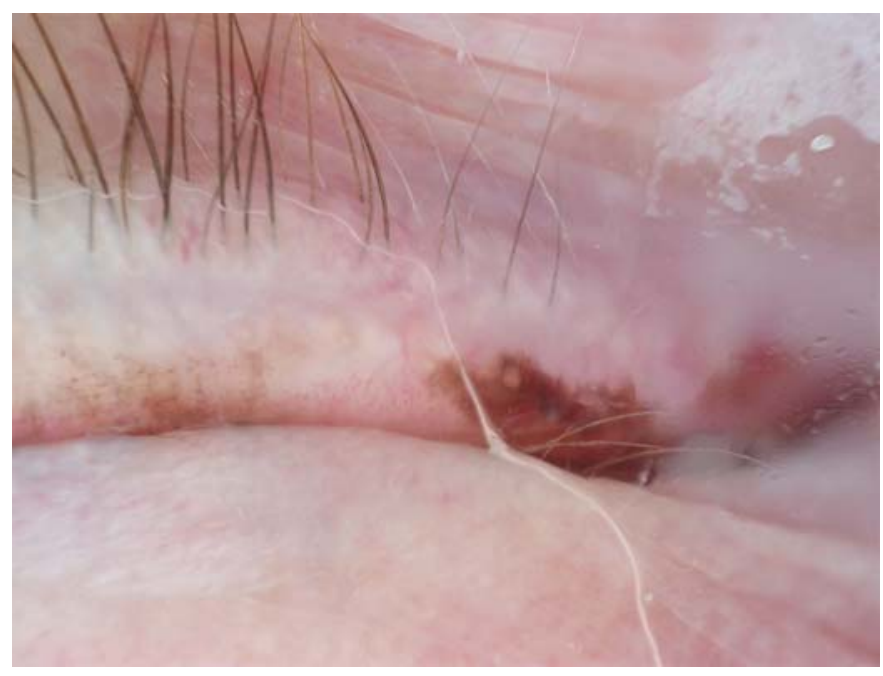

Fig. 3. Red and gray colours were present mostly in the tarsal conjunctiva. Vessels with a rope-ladder pattern (scar-like) were found.

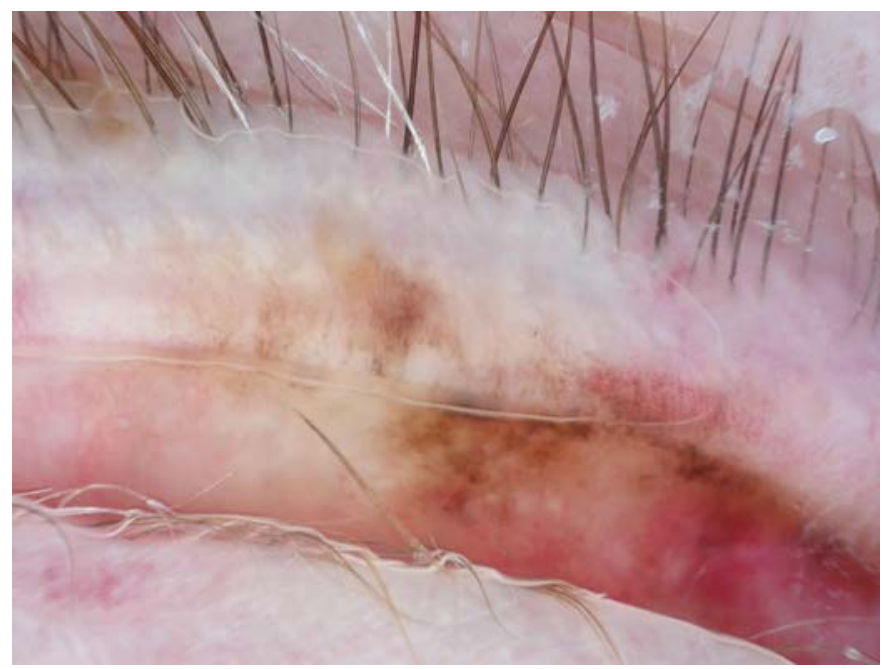


Fig. 4. Histopathological examination revealed atypical proliferation of melanocytes in a lentiginous pattern. Melanocytes showed a pagetoid distribution between the keratinocytes in the epithelium.

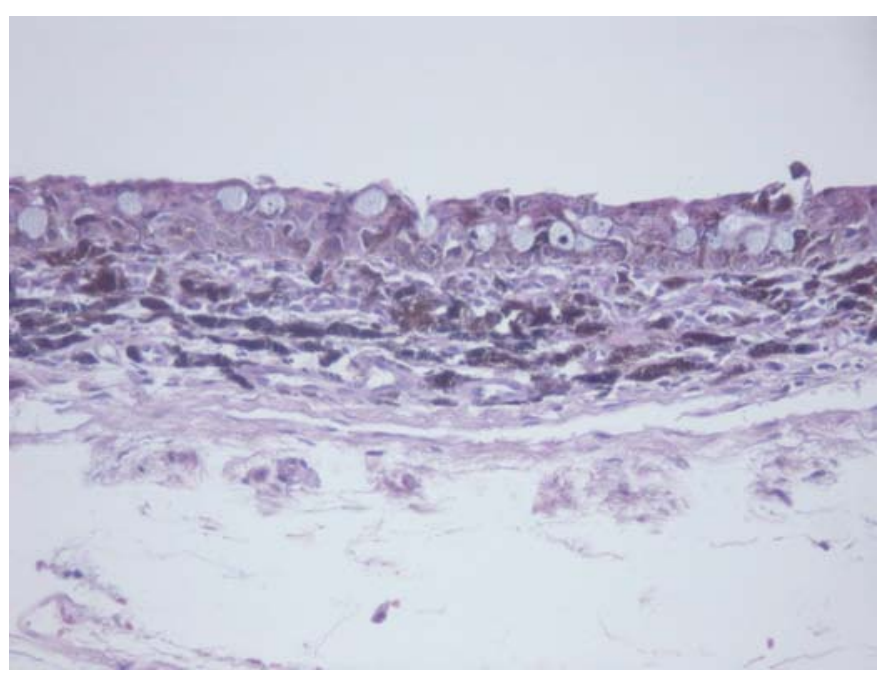

Fig. 5. Melanocytic marker Melan-A expression was consistent with LM in immunohistochemical studies.

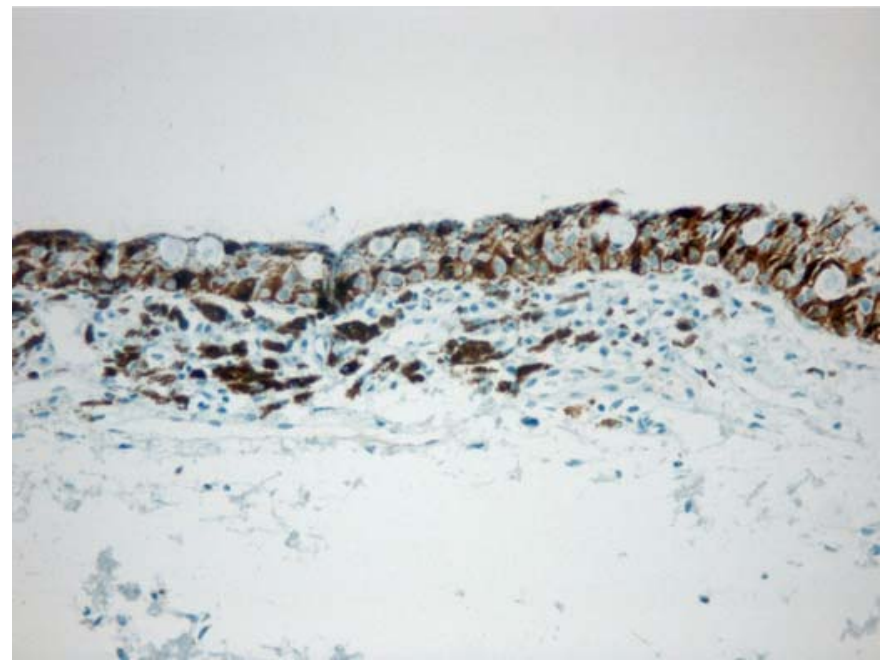


Fig. 6. One-year follow-up showed the patient was disease-free.

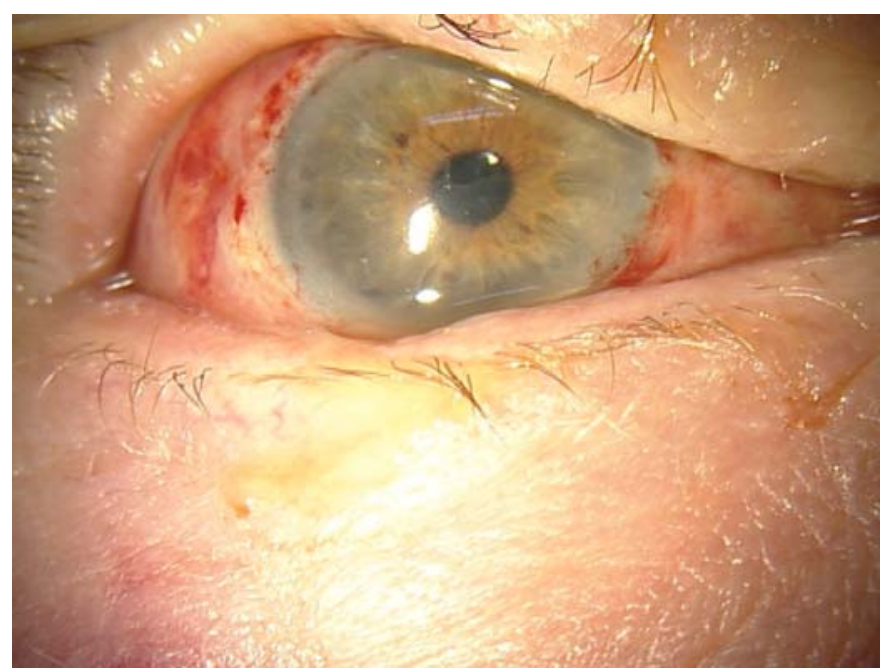




\section{References}

1 Collin JR, Garner A, Allen LH, Hungerford JL: Malignant melanoma of the eyelid and conjunctiva. Aust N Z J Ophtalmol 1986;14:29-34.

2 Werschnik C, Lommatzsch PK: Long-term follow-up of patients with conjunctival melanoma. Am J Clin Oncol 2002;25:248-255.

-3 Saornil MA, Becerra E, Méndez MC, Blanco G: Conjunctival tumors. Arch Soc Esp Oftalmol 2009;84:7-22.

4 Shields $\mathrm{Cl}$, Shields JA: Ocular melanoma: relatively rare but requiring respect. Clin Dermatol 2009;27:122-133.

-5 Grin JM, Grant-Kels JM, Grin CM, Berke A, Kels BD: Ocular melanomas and melanocytic lesions of the eye. J Am Acad Dermatol 1998;38:716-730.

6 Farber M, Schutzer P, Mihm MC Jr: Pigmented lesions of the conjunctiva. J Am Acad Dermatol 1998;38:971-978.

7 Tosy GM, Rubegni P, Schuerfeld K, Toti P: Digital surface microscopy analysis of conjunctival pigmented lesions: a preliminary study. Melanoma Res 2004;14:375380 .

-8 Damato B, Coupland SE: Conjunctival melanoma and melanosis: a reappraisal of terminology, classification and staging. Clin Experiment Ophthalmol 2008;36:786-795.

9 Maly A, Epstein D, Meir K, Pe'er J: Histological criteria for grading of atypia in melanocytic conjunctival lesions. Pathology 2008;40:676-681.

10 Junkins-Hopkins JM: Imiquimod use in the treatment of lentigo maligna. J Am Acad Dermatol 2009;61:865-867.

11 Murchinson AP, Washington CV, Soloman AR, Bernardino CR: Ocular effects of imiquimod with treatment of eyelid melanoma in situ. Dermatol Surg 2007;33:1136-1138. 